\title{
SYSTEMIC SCLEROSIS WITH NEUROLOGICAL MANIFESTATION: NEUROMYELITIS OPTICA
}

\author{
Francisco Alves Bezerra Neto ${ }^{1, \star}$, Artur Diógenes Freitas ${ }^{1}$, Manuel Moreira Neto $^{1}$, Francisco Deoclécio Damasceno Rocha² \\ 1.Universidade Federal do Rio Grande do Norte, Natal (RN), Brazil; 2.Universidade Potiguar, Natal (RN), Brazil. \\ *Corresponding author: bezeneto@hotmail.com
}

\section{BACKGROUND}

The authors describe a case of a patient with a previous diagnosis of systemic sclerosis who developed neuromyelitis optica.

\section{CASE REPORT}

Female, 50 years old, diagnosed with systemic sclerosis, exhibiting sclerodactyly, telangiectasias of the hands, Raynaud's phenomenon, ANA 1/1280 centromere pattern and transthoracic echocardiogram with pulmonary hypertension. Six months after diagnosis, she developed paresthesia up to the level of the umbilical region, difficulty walking associated with urinary retention and constipation, and was admitted for investigation of the condition. At the time, she was taking: 15 mg/week methotrexate, 5 $\mathrm{mg}$ folic acid and $30 \mathrm{mg}$ nifedipine. In the neurological examination on admission, the patient had reduced vibratory and pain sensitivity below the level of T10 on the right side and painful hypoesthesia between T8-T10 on the left, grade 5 strength in the upper and lower limbs, deep reflexes in the upper limbs, exalted activity in patellar and Achilles bilaterally, and cutaneous-plantar in extension. Cerebrospinal fluid (CSF) analysis showed cellularity of $22 \mathrm{~mm}^{3}$ ( $96 \%$ mononuclear), $79.2 \mathrm{mg} / \mathrm{dL} \mathrm{proteins}, 60 \mathrm{mg} / \mathrm{dL}$ glucose, nonreactive VDRL and negative AFB. Neuroaxis magnetic resonance showed multiple hyperintense lesions on T2 in the brain, with no expansive effect, dispersed in the deep/subcortical white matter of the cerebral hemispheres, suggesting lesions with demyelinating substrate. In the cervical spine, there were hyperintense spinal cord lesions on T2 at the level of C1 to C2 and from $\mathrm{C} 3$ to $\mathrm{C} 7$, the latter longitudinally extensive with a slight tumefactive effect. Finally, in the thoracic spine, the involvement was intramedullary lesions, centrally located and longitudinally extensive in the topography from T3 to T6 and T7 to T10. A test for 1/40 anti-aquaporin 4 antibody was conducted. Based on clinical and laboratory data, we diagnosed neuromyelitis optica associated with systemic sclerosis. Pulse therapy with $1 \mathrm{~g}$ methylprednisolone was carried out for three days, associated with $1 \mathrm{~g}$ rituximab in weeks 0 and 2. The patient's paresthesia improved significantly, as did her ability to walk and total sphincter control.

\section{CONCLUSION}

Although neuromyelitis optica is known to coexist with other autoimmune disorders (e.g., SLE, Sjögren's), it has no prior relationship with systemic sclerosis. This association is rarely reported in the literature and it is not yet fully understood whether systemic sclerosis is an etiological factor in neuromyelitis optica. The CNS involvement is also rare in systemic sclerosis, but there are reports of headache, seizure, and cognitive impairment.

\section{KEYWORDS}

Systemic sclerosis, Neurological, Neuromyelitis optica. 